\title{
Bright On-Demand Source of Antibunched Microwave Photons Based on Inelastic Cooper Pair Tunneling
}

\author{
A. Grimm, ${ }^{1,}{ }^{*}$ F. Blanchet, ${ }^{1}$ R. Albert, ${ }^{1}$ J. Leppäkangas, ${ }^{2}$ S. Jebari, ${ }^{1}$ D. Hazra, ${ }^{1}$ F. Gustavo, ${ }^{1}$ \\ J.-L. Thomassin, ${ }^{1}$ E. Dupont-Ferrier, ${ }^{3}$ F. Portier, ${ }^{4}$ and M. Hofheinz ${ }^{1,5, \uparrow}$ \\ ${ }^{1}$ Université Grenoble Alpes, CEA, INAC-PHELIQS, F-38000 Grenoble, France \\ ${ }^{2}$ Physikalisches Institut, Karlsruhe Institute of Technology, 76131 Karlsruhe, Germany \\ ${ }^{3}$ Institut Quantique/Département de physique, Université de Sherbrooke, \\ Sherbrooke, Québec JIK 2R1, Canada \\ ${ }^{4}$ SPEC, CEA, CNRS, Université Paris-Saclay, CEA-Saclay 91191 Gif-sur-Yvette, France \\ ${ }^{5}$ Institut Quantique/Département GEGI, Université de Sherbrooke, Sherbrooke, Québec J1K 2RI, Canada
}

(Received 12 October 2018; revised manuscript received 15 January 2019; published 24 April 2019)

Single-photon generation is an important proof of the underlying quantum nature of a physical process and a ubiquitous tool for scientific exploration, with applications ranging from spectroscopy and metrology to quantum computing. In the microwave regime, emission of antibunched radiation has so far relied on coherent control of Josephson qubits requiring precisely calibrated microwave pulses. In this work, we experimentally demonstrate the operation of a bright on-demand source of quantum microwave radiation driven by a simple dc voltage bias across a Josephson junction. Our source is based on photon emission into a microwave resonator through inelastic Cooper pair tunneling regulated by a high-impedance $R C$ circuit preventing simultaneous tunnel events. It is characterized by its normalized second-order correlation function of $g^{(2)}(0) \approx 0.43$ corresponding to antibunching in the single-photon regime. Our device can be triggered, and its in situ tunable emission rate exceeds those obtained with current microwave singlephoton sources by more than 1 order of magnitude.

DOI: 10.1103/PhysRevX.9.021016

\section{INTRODUCTION}

Josephson photonics has recently emerged as a way to directly generate and manipulate quantum microwaves without the need for complicated microwave control drives [1-8]. It relies on inelastic Cooper pair tunneling where a dc-voltage-biased Josephson tunnel junction can admit a finite direct current, even if the applied bias voltage is smaller than its gap [9]. Although the finite voltage bias makes it impossible for Cooper pairs to tunnel elastically in this regime, they can tunnel inelastically while dissipating their surplus energy into the electromagnetic environment of the junction. The resulting excitations of the environment are photons at microwave frequencies [10,11]. This effect can be harnessed, and the properties of the emitted

\footnotetext{
*alexander.grimm@yale.edu

†max.hofheinz@usherbrooke.ca
}

Published by the American Physical Society under the terms of the Creative Commons Attribution 4.0 International license. Further distribution of this work must maintain attribution to the author(s) and the published article's title, journal citation, and DOI.
Subject Areas: Mesoscopics, Photonics, Quantum Physics

radiation can be controlled by presenting the junction with a specifically tailored electromagnetic environment.

In particular, a Cooper pair of charge $2 e$ can tunnel inelastically through a Josephson junction biased at voltage $V_{\mathrm{b}}$ if the electromagnetic environment of the junction has modes that can absorb the energy difference of $2 e V_{\mathrm{b}}=$ $h \nu_{\mathrm{J}}=\sum_{i} n_{i} h f_{i}$, where $\nu_{\mathrm{J}}=2 e V_{\mathrm{b}} / h$ is the Josephson frequency and $n_{i}$ is the number of photons emitted into a given mode of frequency $f_{i}$. The power spectral density (PSD) in units of photons of the emitted radiation at frequency $f$ is then given by the expression [11]

$$
\gamma\left(f, \nu_{\mathrm{J}}\right)=\frac{I_{\mathrm{c}}^{2}}{2} \frac{\operatorname{Re} Z(f)}{f} P\left(h \nu_{\mathrm{J}}-h f\right) .
$$

Here, $I_{\mathrm{c}}$ is the critical current of the junction, $\operatorname{Re} Z(f)$ is the real part of the impedance describing the electromagnetic environment, and $P(h f)$ is the probability density for a tunneling Cooper pair to exchange an energy $h f$ with this environment $[9,12]$. The latter quantity is directly proportional to the Cooper pair tunneling rate $\Gamma$ :

$$
\Gamma\left(\nu_{\mathrm{J}}\right)=\frac{I_{\mathrm{c}}^{2}}{4} \frac{h}{4 e^{2}} P\left(h \nu_{\mathrm{J}}\right) .
$$


While early experiments focused on the rate $\Gamma$ and the associated direct current [10,13-15], recent advances in low-noise high-frequency measurements have made it possible to investigate the photonic side of this energy transfer $[6-8,11,16]$. In all previous implementations, the tunneling events happened either independently $[10,11]$ or through stimulated emission [6-8], in both cases leading to classical Poisson statistics of the emitted photons. The present work, as well as Ref. [17], demonstrates a device where the electromagnetic environment of the junction is engineered to create antibunched photon emission, thus showing quantum statistics generated through inelastic Cooper pair tunneling.

\section{DEVICE AND WORKING PRINCIPLE}

A schematic circuit diagram of our device is shown in the bottom part of Fig. 1(a). Its main element is a superconducting quantum interference device (SQUID, yellow) made of two parallel $0.02 \mu \mathrm{m}^{2} \mathrm{NbN}-\mathrm{MgO}-\mathrm{NbN}$ Josephson junctions [18] [Fig. 1(d)]. NbN was chosen because of its large superconducting gap, which allows, in principle, for operating frequencies of up to approximately $1 \mathrm{THz}$ for Josephson photonics devices [19]. Alternatively, our device could be implemented in a standard $\mathrm{Al}-\mathrm{AlO}_{x}$-based fabrication process for straightforward integration with circuit quantum electrodynamics systems. We use a fast flux line to tune its critical current and thus the tunneling rate in situ. One side of the SQUID connects to a quarter-wave transmission line resonator [blue in Fig. 1(a)], with a fundamental frequency $f_{0}=6 \mathrm{GHz}$, which is followed by an on-chip bias $\mathrm{T}$ and beam splitter [Figs. 1(a), 1(b) and Ref. [19]]. The other side of the SQUID is grounded through an on-chip parallel $R C$ circuit (red), with resistance $R$, capacitance $C$, and a small spurious inductance $L_{\mathrm{p}}$ [19].

We extract the calibrated and time-resolved autocorrelations and crosscorrelations $\left(G^{(1)}, G^{(2)}\right)[5,20,21]$, as well as the power spectral density (PSD) of the microwave radiation emitted by our device using the measurement setup depicted schematically in Fig. 1(a) and described in detail in Ref. [19]. The PSD divided by $h f$ has units of photons and corresponds to the quantity computed by Eq. (1).

The underlying principle of our source is that one tunneling event necessarily acts back onto the next one in order to create anticorrelations in the Cooper-pair current, leading to antibunching in the photon emission. On timescales shorter than $R C$, a tunneling Cooper pair has to charge the island formed between the capacitor and SQUID. To emit a photon, the voltage bias must be chosen such that $h \nu_{\mathrm{J}}=h f_{0}+E_{\mathrm{C}}$, where $E_{\mathrm{C}}=(2 e)^{2} / 2 C$ is the charging energy of the island [Fig. 1(e)]. However, immediately after a first tunneling event, the energy necessary to add another Cooper pair to the island is $(2 \cdot 2 e)^{2} / 2 C-(2 e)^{2} / 2 C=3 E_{\mathrm{C}}$, which makes the voltage

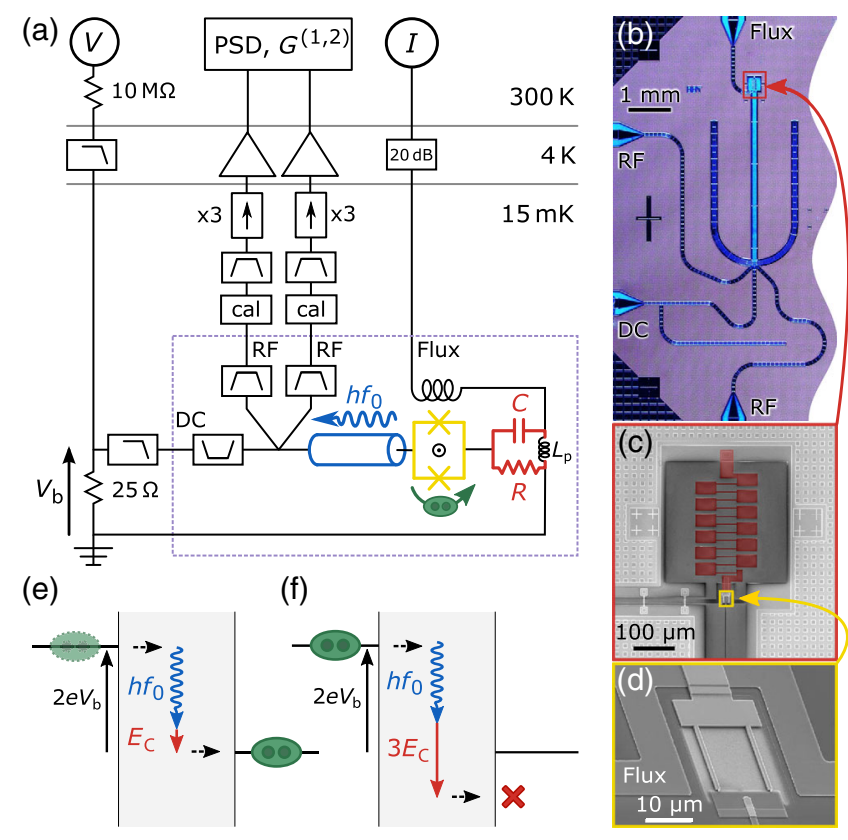

FIG. 1. Sample, setup, and working principle. (a) Overview of the sample and measurement setup. The SQUID (yellow) is connected to an $R C$ element (red, with parasitic inductance $L_{\mathrm{p}}$ ) and a transmission line resonator with a fundamental mode at $f_{0}$ (blue). Voltage and flux biases are applied to the SQUID through a voltage divider and an on-chip fast flux line, respectively. Photons escape from the resonator through an on-chip bias $\mathrm{T}$ and beam splitter into two measurement chains, each containing a calibration assembly [19], as well as filters and isolators protecting the sample from amplifier noise. PSD and the photon correlation functions $G^{(1)}$ and $G^{(2)}$ are calculated numerically after down-mixing and digitization [19]. (b) Optical image overview of the chip [framed part of (a)]. (c) Scanning electron micrograph (SEM) of the SQUID (small yellow frame) and $R C$ circuit (red). (d) SEM of the SQUID consisting of two vertical NbN-MgO-NbN Josephson junctions, surrounded by the fast flux line. Their crossover forms the capacitor of the $R C$ element. (e) Inelastic tunneling process. Horizontal black lines represent the Cooper-pair condensates on either side of the barrier (gray) of the voltage-biased SQUID. The voltage bias $\left(2 e V_{\mathrm{b}}=h f_{0}+E_{\mathrm{C}}\right)$ is chosen to enable tunneling with photon emission at $f_{0}$ when the capacitor is not already charged. (f) Immediately after a tunneling event, the energy necessary to charge the capacitor with another Cooper pair is $3 E_{\mathrm{C}}$, blocking further Cooper pair tunneling for a time of approximately $R C$.

bias insufficient for further tunneling with photon emission [Fig. 1(f)]. A second Cooper pair can tunnel and emit a photon only after a time $R C$, when the island is discharged, leading to the desired antibunching. This picture is valid if the charging energy associated with one Cooper pair is large compared to the thermal energy fluctuations, i.e., $E_{\mathrm{C}} \gg$ $k_{\mathrm{B}} T_{\text {eff }}$, as well as the lifetime broadening of the charging energy, i.e., $E_{\mathrm{C}} \gg \hbar /(2 R C)$, or equivalently $R \gg h /\left(4 e^{2}\right) \approx$ $6.5 \mathrm{k} \Omega$. Here, $T_{\text {eff }}$ is the effective temperature of the lowfrequency electromagnetic environment [19]. 


\section{DEVICE PARAMETERS AND PSD OF THE EMITTED RADIATION}

We now verify these conditions and extract the device parameters. To this end, we analyze the PSD in Fig. 2(a) taken at a flux bias $\Phi \approx 0.5 \Phi_{0}$, where $\Phi_{0}=(h / 2 e)$ is the superconducting flux quantum. There, $I_{\mathrm{c}}(\Phi)$ is small and thus emission rates are low. Hence, tunneling events can be considered independent and Eqs. (1) and (2) are valid [9]. For this flux bias, photon emission is maximal at $\nu_{\mathrm{J}}=$ $7.5 \mathrm{GHz}$ and $f=f_{0}=6 \mathrm{GHz}$. The difference between the energy $h \nu_{\mathrm{J}}$ provided by a tunneling Cooper pair and the energy $h f$ of the detected photons is the charging energy $E_{\mathrm{C}} \approx h \times 1.5 \mathrm{GHz} \approx k_{\mathrm{B}} \times 72 \mathrm{mK} \quad(C \approx 51 \mathrm{fF})$. Further analysis of this PSD [19] yields the real part of the resonator impedance [blue dots in Fig. 2(b)], and the effective temperature of the low-frequency electromagnetic environment $T_{\text {eff }} \approx 21 \mathrm{mK}$. The resistance $R \approx 32.1 \mathrm{k} \Omega$ was determined from an independent dc measurement [19]. Additionally, we verify the accuracy of the electrical model presented in Fig. 1(a) by using it to fit Eq. (1) to the PSD. The real part of the resonator impedance given by the resulting circuit parameters is shown as a solid green line in
Fig. 2(b) (for detailed parameters, see Ref. [19]). It has a full width at half maximum of $575 \mathrm{MHz}$ and agrees well with the measured data. The approximately $200-\mathrm{MHz}-$ periodic impedance modulations are due to reflections in our output lines, which are not included in the model.

In Fig. 2(c), we explore the behavior of the device when the critical current is increased to values relevant for operation, where Cooper pair tunneling events cannot be considered independent anymore. To do so, we measure the power spectral density at its maximal value in frequency (at $f_{0}$ ) as a function of $\nu_{\mathrm{J}}$ and the flux bias $\Phi$. The brightest features appear around $\nu_{\mathrm{J}}=7.5 \mathrm{GHz}$, corresponding to the desired process. At slightly lower flux bias, additional features appear at $\nu_{\mathrm{J}}=13.5 \mathrm{GHz}$. They originate from the emission of two photons per tunneling Cooper pair into the mode at $f_{0}$. When the critical current is maximal $(\Phi=0)$, another feature becomes visible at $\nu_{\mathrm{J}}=25.4 \mathrm{GHz}$. It corresponds to processes where one Cooper pair emits one photon into mode $f_{0}$ and one into the next mode of the quarter-wave transmission line resonator at $f_{1} \approx 3 f_{0}$.

Strikingly, the processes at 7.5 and $13.5 \mathrm{GHz}$ disappear around $\Phi=0$, where one would expect the rates to be

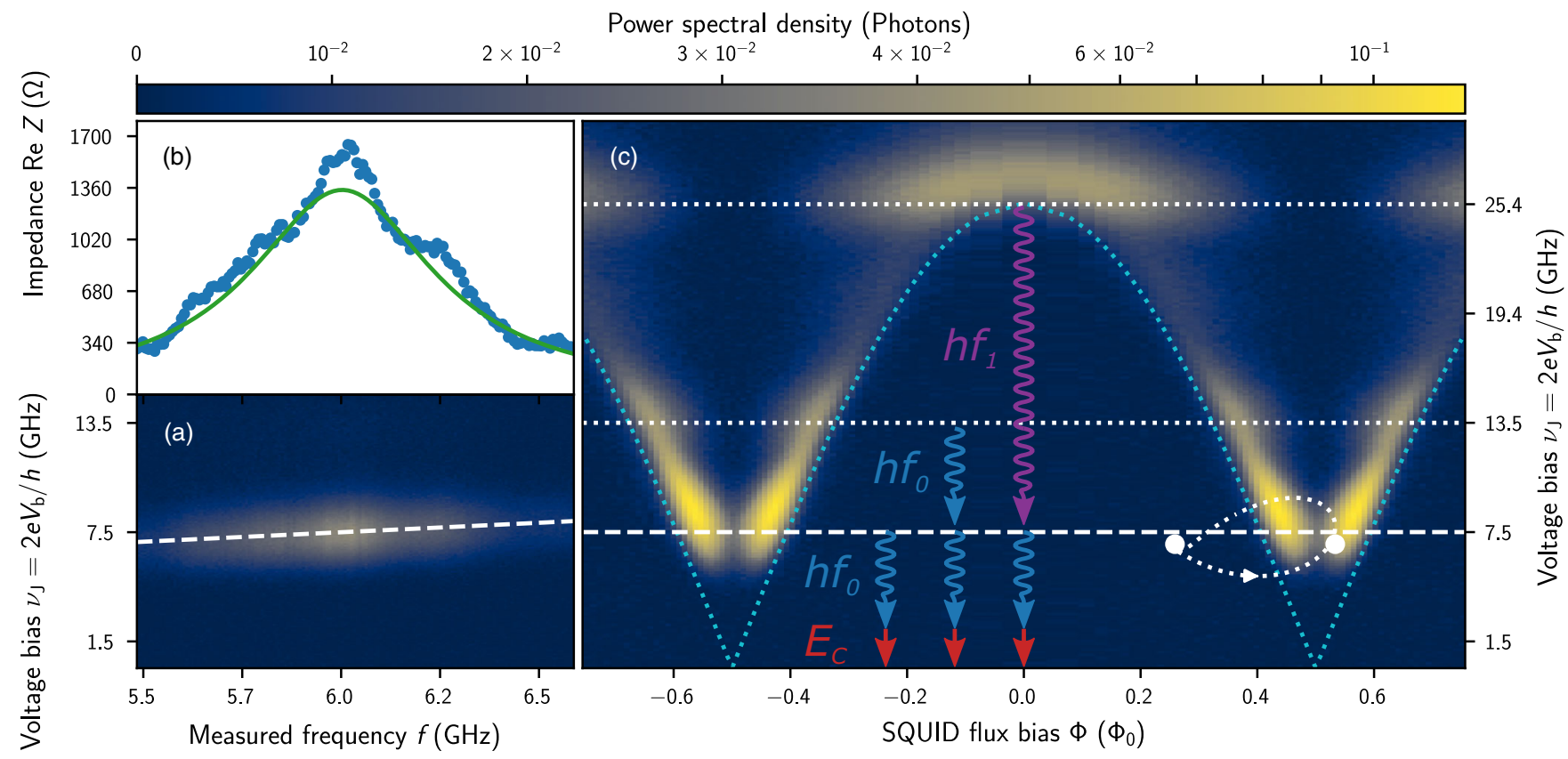

FIG. 2. (a) Power spectral density in photon units measured close to minimal $I_{\mathrm{c}}(\Phi)$ (SQUID flux bias $\Phi \approx 0.5 \Phi_{0}$ ) as a function of frequency and voltage bias (expressed as Josephson frequency $\nu_{\mathrm{J}}$ ). The dashed line corresponds to the process $h \nu_{\mathrm{J}}=E_{\mathrm{C}}+h f$, with $E_{\mathrm{C}} \approx 1.5 \mathrm{GHz}$ the energy required to charge the capacitor with one Cooper pair. Photon emission is highest for $f \approx f_{0} \approx 6 \mathrm{GHz}$, the resonance frequency of the transmission line resonator. (b) Real part of the impedance as a function of voltage bias extracted from the data shown in panel (a) (blue dots) and plotted from a fit of our circuit model to the data (green line). (c) Power spectral density in photon units at $6 \mathrm{GHz}$ as a function of voltage and flux biases. The horizontal lines and sketches indicate the one-photon process $h \nu_{\mathrm{J}}=$ $E_{\mathrm{C}}+h f_{0}$ (dashed line) and the two-photon processes $h \nu_{\mathrm{J}}=E_{\mathrm{C}}+2 h f_{0}$ and $h \nu_{\mathrm{J}}=E_{\mathrm{C}}+h f_{0}+h f_{1}$ (dotted lines), where $f_{1}$ is the frequency of the next resonator mode. Values of $E_{\mathrm{C}}$ and $f_{0}$ are extracted from panel (a) and match the observed features. The turquoise dotted curve delimits the region where no voltage bias can be applied because the SQUID latches to its current branch (see text). Two large white dots mark the starting and ending points of the flux pulse giving the results of Fig. 4. The dotted loop indicates the estimated trajectory. 
highest. We instead observe a dark zone delimited by the turquoise dotted line. In this region, the critical current is high enough for the SQUID to get trapped in a Bloch oscillation regime [22-25], a rudiment of the zero-voltage state observed in larger Josephson junctions. In this state, the voltage drops mostly over the $R C$ element, decreasing the voltage difference across the SQUID below the threshold for photon emission at $f_{0}$. This interpretation is confirmed by an independent measurement of the resonator frequency showing flux tunability in the region delimited by the dotted line [19].

\section{FREE-RUNNING MODE OF OPERATION}

We now focus on the key question of this work and investigate the statistics of the radiation emitted by the device. To do so, we measure its normalized second-order correlation function $g^{(2)}(t, \tau)$ given by [26]

$$
g^{(2)}(t, \tau)=\frac{G^{(2)}(t, \tau)}{G^{(1)}(t, 0) G^{(1)}(t+\tau, 0)} .
$$

In the above expression, $G^{(2)}(t, \tau)$ is the unnormalized second-order correlation function, which is dependent on a time $t$ with respect to a reference and on the time delay $\tau$, defined as

$$
G^{(2)}(t, \tau)=\left\langle\hat{a}_{\text {out }}^{\dagger}(t) \hat{a}_{\text {out }}^{\dagger}(t+\tau) \hat{a}_{\text {out }}(t+\tau) \hat{a}_{\text {out }}(t)\right\rangle .
$$

The operators $\hat{a}_{\text {out }}$ and $\hat{a}_{\text {out }}^{\dagger}$ are the annihilation and creation operators of the outgoing field in the transmission line. The denominator of the right-hand side of Eq. (3) is a normalization factor dependent on the first-order correlation function

$$
G^{(1)}(t, \tau)=\left\langle\hat{a}_{\text {out }}^{\dagger}(t) \hat{a}_{\text {out }}(t+\tau)\right\rangle .
$$

Here, $G^{(1)}(t, 0)$ gives the instantaneous photon emission rate of our device. In the absence of a well-defined time reference, an average over $t$ is performed on $G^{(1)}(t, \tau)$ and $G^{(2)}(t, \tau)$, yielding $G^{(2)}(\tau), G^{(1)}(\tau)$ and $g^{(2)}(\tau)$ [26].

Figure 3(a) shows the total photon emission rate $G^{(1)}(0)$ of our sample in a region around the one-photon peak visible in Fig. 2(c). It is measured by integrating the PSD over frequency between 4.22 and $8 \mathrm{GHz}$ [19]. We have evaluated $g^{(2)}(\tau)$ at different points along two lines of constant voltage (flux) bias as indicated by plus (cross) symbols, corresponding to the curves shown in Fig. 3(b) [Fig. 3(c)].

Close to $\Phi \approx 0.5 \Phi_{0}$, the $g^{(2)}(\tau)$ function displays a marked dip down to approximately 0.5 at $\tau=0$ and is close to the expected value of 1 elsewhere, showing that our sample indeed produces antibunched photons. Figure 3(b) shows that, as we approach lower flux biases to increase the critical current, the sharp dip close to $\tau=0$ remains, but a broad bunching peak develops around it. We attribute this broad peak to stochastic jumps between the bright voltage

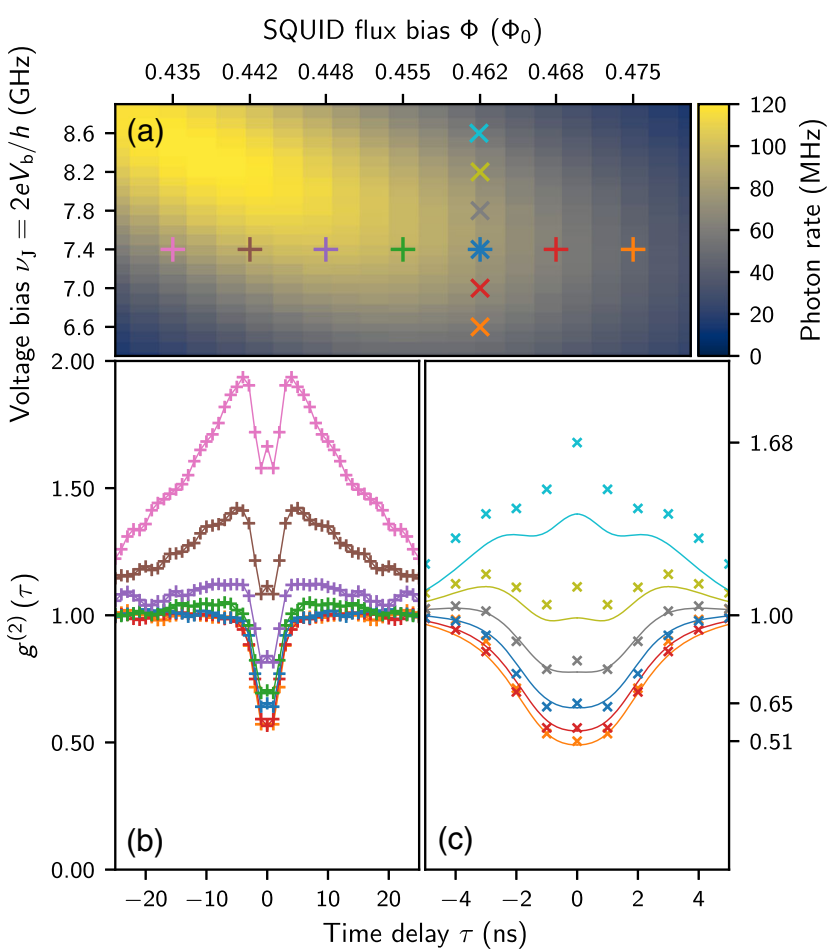

FIG. 3. Emission rate and statistics. (a) Photon emission rate measured around the one-photon peak from 4.22 to $8 \mathrm{GHz}$. Symbols mark the points where the data shown in panels (b) and (c) were taken. (b) Second-order correlation functions at voltage bias $\nu_{\mathrm{J}}=7.4 \mathrm{GHz}$ for different flux biases. Lines are a guide for the eye. For clarity, error bars are omitted here and in panel (c) $(3 \sigma \approx 0.1$, see Ref. [19]). Curves from top to bottom correspond to the + symbols in panel (a) from left to right. (c) Second-order correlation functions at flux bias $\Phi=0.462 \Phi_{0}$ for different voltage biases. Curves from top to bottom correspond to the $\times$ symbols in panel (a) from top to bottom. Higher voltage biases enable multiple tunneling events and thus bunching. Solid lines are numerical simulations following Ref. [27] (see text).

state of the junction and the dark zero-voltage state discussed above. This interpretation is consistent with the reduction in emission rate towards the lower-left corner of Fig. 3(a). The width of the bunching peak $(\approx 20 \mathrm{~ns})$ then corresponds to the switching time between the bright and dark states. The persistence of the sharp dip at $\tau=0$ demonstrates that the intended blocking mechanism remains functional, despite this effect.

Figure 3(c) shows the sensitivity of the antibunching to precise adjustment of the bias voltage. Here, we choose the flux bias where we achieve the highest photon rate, while bunching due to jumps to the dark state remains negligible: $\Phi \approx 0.462 \Phi_{0}$. When biased above $8 \mathrm{GHz}$, our sample emits bunched light $\left[g^{(2)}(0)>1\right]$. This bunching effect can be understood by considering the energy diagrams in Figs. 1(e), 1(f) at higher voltage biases: Even though the voltage is initially too high for the resonance condition to be fulfilled, thermal fluctuations still occasionally allow Cooper pairs to tunnel. When a first Cooper pair does 
tunnel, the $R C$ circuit is charged, and a second one can more easily follow, leading to bunching. As the bias decreases, so does the value of the second-order correlation function, down to $g^{(2)}(0) \approx 0.5 \pm 0.1( \pm 3 \sigma)$. The lowest value of $g^{(2)}(0)$ is reached below the maximum of the photon emission rate, where residual double emission events caused by thermal fluctuations of the bias voltage are further suppressed. The blue curve is taken at the maximum emission rate of $77 \mathrm{MHz}\left(\nu_{\mathrm{J}}=7.4 \mathrm{GHz}\right)$ along this cut. At this point, we measure $g^{(2)}(0) \approx 0.65 \pm 0.06$.

The solid lines in Fig. 3(c) are numerical calculations up to fourth order in the critical current [27,28] using the electrical model of Fig. 1(a) with the extracted device parameters and an effective temperature of $T_{\text {eff }}=40 \mathrm{mK}$. They reproduce well the observed antibunching signatures at the lowest bias voltages, including bumps in $g^{(2)}$ at approximately $2 \mathrm{~ns}$, but they fail to fully explain the bunching signatures for the highest bias voltages. In addition, $T_{\text {eff }}$ used here to reproduce the data is significantly higher than the temperature of the electromagnetic environment extracted from the data in Fig. 2(a) at low critical current and at low bias voltage. This discrepancy could indicate that the resistor of the $R C$ element heats up more than expected at the higher photon fluxes and higher voltages used here. Another explanation could be that correlations between more than two Cooper pairs are relevant and that calculations have to be performed beyond fourth order in the critical current, which significantly increases the computational effort and is left for future work.

We conclude that antibunching is robust as long as the bias voltage is kept at the nominal resonant value or below, but it is rapidly transformed into bunching above. Double emission events could be further suppressed in a future iteration of the device by increasing the charging energy while keeping the $R C$ time constant. A simultaneous increase of the emission frequency would move the relevant peaks away from the dark region to higher voltage biases and thus avoid a trade-off between improved antibunching and emission rate. The value of $g^{(2)}(0)$ could be further decreased by increasing the separation between the characteristic time of the $R C$ element $(R C \approx 1.64 \mathrm{~ns})$ and the lifetime of the resonator $\left(\tau_{\mathrm{r}} \approx 0.28 \mathrm{~ns}\right)$.

\section{PULSED MODE OF OPERATION}

So far, we have focused on the free-running mode of operation, where latching to the dark state prevents us from reaching higher emission rates. However, as we now show, we can make use of this effect to produce photons on demand through the flux pulsing scheme indicated in white on Fig. 2(c). For this case, the voltage bias is set to its nominal value $\left(\nu_{\mathrm{J}}=7.4 \mathrm{GHz}\right)$. We start out at a flux bias well in the dark region (left white dot), where no photon emission occurs in the stationary regime. We then apply a flux pulse that frustrates the SQUID (right white dot), suppressing its critical current and resetting it to the voltage branch. At this bias point, photon emission is unlikely. Returning to the initial point in the dark region removes the frustration and allows a photon to be emitted (and the capacitor to be charged). Then, the system is again trapped in the dark state, limiting photon emission to at most one per cycle.

Figure 4 shows the data obtained when Gaussian flux bias pulses with FWHM of 1.5 ns are applied every 6 ns, corresponding to a repetition rate of approximately $167 \mathrm{MHz}$. Different pulse durations (within a factor of 2) and lower repetition rates do not affect the results significantly. Higher repetition rates, however, cause photon pulses to overlap, and significantly shorter pulses would have frequency components at the emission frequency of our device.

In Fig. 4(a), we first explore the instantaneous photon emission rate $G^{(1)}(t, 0)$ as a function of time $t$ with respect to the pulse. One averaged measurement yields the values marked by blue dots, which are separated by the sampling period of $1 \mathrm{~ns}$ of our measurement. By shifting the time delay between pulse generation and the beginning of the sampling window, $G^{(1)}(t, 0)$ can be resolved below the sampling period, yielding the orange curve in Fig. 4(a).

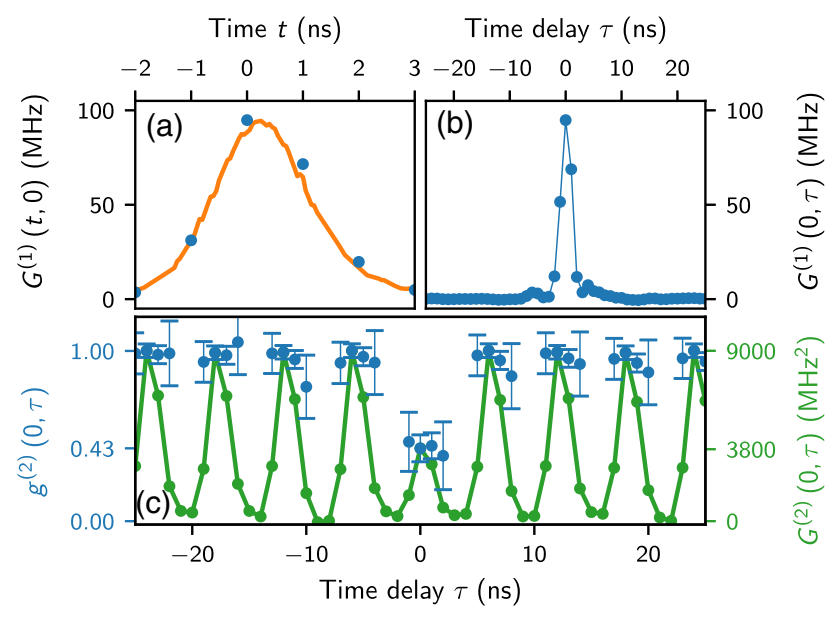

FIG. 4. Flux-pulsed on-demand operation. (a) Instantaneous photon emission rate as a function of time. Blue dots are the rates acquired during one averaged measurement; the orange line is obtained by repeating the measurement with different time delays of the flux pulse. The shape of the peak is slightly skewed due to the finite time the photon spends in the resonator. The statistical errors in this panel and the next are small compared to the data points [19]. (b) First-order correlation function at $t=0$, as a function of the time delay $\tau$ (the solid line is a guide for the eye). As the actual time of emission is slightly after $t=0$, the peak is centered at slightly positive $\tau$. (c) Second-order correlation function at $t=0$, as a function of the time delay $\tau$. The green dots correspond to the unnormalized second-order correlation function (right ordinate) and display periodic peaks every $6 \mathrm{~ns}$ given by the period of the applied flux pulse (the line is a guide for the eye). Blue dots with error bars $( \pm 3 \sigma)$ show the normalized correlation function (left ordinate). 
The average of $G^{(1)}(t, 0)$ over one pulse period gives the emission rate $(\approx 33 \mathrm{MHz})$, meaning that our source emits approximately 0.2 photons per pulse. The zero on the time axis matches the time $t=0$ at which the measurements in Figs. 4(b), 4(c) were taken. The shape of this peak differs from the ideal single-sided exponential decay because the frequency profile of our broadband cascaded transmission line resonator is only approximately Lorentzian [see Fig. 2(b)], and its edges are removed through filtering [19]. In addition, we expect uncertainties in the timing of the tunneling event with respect to the time reference (jitter) due to the finite rise time of the flux pulse.

We can get a rough estimate of the relative importance of jitter in the width of $G^{(1)}(t, 0)$ by comparing with the complementary measurement of $G^{(1)}(0, \tau)$ [Fig. 4(b)], which characterizes the first-order coherence of the photons emitted by our source. The width of the peak is very close to the peak in $G^{(1)}(t, 0)$ in Fig. 4(a), indicating that the broadening of $G^{(1)}(t, 0)$ is not dominated by jitter, a necessary (but not sufficient) condition for indistinguishable photons. Also note that $G^{(1)}(0,6 \mathrm{~ns}) \approx 0$, showing that there is no coherence between successive pulses as expected for single photons.

In the unnormalized second-order correlation function $G^{(2)}(0, \tau)$, this picture is reversed [Fig. 4(c)]. Peaks appear every $6 \mathrm{~ns}$, equal to the period of the applied flux pulse, proving the on-demand aspect of our source. Note that the peak at $\tau=0$ is significantly lower than the others, indicating the antibunched character of the emitted radiation.

In order to quantify the antibunching, we compute the normalized second-order correlation function (blue dots) according to Eq. (3). This computation is only done for times $\tau$ where such a normalization is meaningful, i.e., where the emission rate $G^{(1)}(0+\tau, 0) \neq 0$. For the remaining points, the bound on the associated error would be larger than the range of the data. For time differences $\tau=n \times 6$ ns with $n \neq 0$, we obtain $g^{(2)} \approx 1$, indicating that photons from two different pulses are independent. At zero time delay, however, $g^{(2)}(0,0)=0.43 \pm 0.08( \pm 3 \sigma)$ : The photons are antibunched, in agreement with the mechanism presented in Figs. 1(e), 1(f).

Here, we achieve stronger antibunching than in the freerunning case, likely due to the additional blocking effect given by the latching to the zero-voltage state in the dark region. At the same time, the pulsing scheme allows us to maintain very good quantum efficiency and photon flux because of the high emission rates at low flux bias. This result makes it likely for a tunnel event to happen during each cycle, even for very short flux pulses. We attribute the residual $g^{(2)}(0,0)$ mainly to the low charging energy of our $R C$ circuit and its relatively low time constant $(R C=1.64 \mathrm{~ns})$, which is comparable to the width of the applied flux pulses (1.5 ns) and only slightly larger than the decay time of the resonator $(0.28 \mathrm{~ns})$. The latter is limited by practical considerations such as the instantaneous bandwidth of our measurement setup [19]. In addition, a parasitic coupling between the flux bias and the $R C$ circuit, described by the parasitic inductance $L_{\mathrm{p}}$ in Fig. 1(a), causes the flux pulse to modulate the voltage across the junction. The dashed white line in Fig. 2(c) indicates the resulting expected trajectory in parameter space, which may not be ideal. Further optimization of this trajectory and modification of the coupling or application of simultaneous voltage and flux pulses could lead to better antibunching. Moreover, this type of device could possibly be optimized as a source of on-demand multiphoton Fock states by addressing the processes appearing at higher bias voltages.

\section{CONCLUSION}

In conclusion, we have demonstrated a Josephson photonics device producing antibunched microwave radiation. In doing so, we have shown that quantum statistics can be generated from inelastic Cooper pair tunneling. By modulating the effective critical current of the SQUID, using fast flux pulses and locking to a dark state after photon emission, we can produce antibunched photons on demand at very high rates. Increasing the charging energy and the $R C$ time, or replacing the $R C$ circuit by a high impedance resonant mode, should allow for significant improvements of antibunching and quantum efficiency in future iterations of the device. We expect that it can be optimized to be an on-demand single-photon source with near unity quantum efficiency, providing an attractive alternative to current devices based on Josephson qubits [29-32]. Such a source could then be used for quantum metrology $[33,34]$, quantum computation with propagating photons [35], or quantum measurements in cases where the shot noise of coherent light needs to be avoided.

\section{ACKNOWLEDGMENTS}

We acknowledge fruitful discussions with the Quantronics Group, B. Kubala, J. Ankerhold, F. Lefloch, and T. Chevolleau, as well as financial support from the Grenoble Nanosciences Foundation (Grant JoQOLaT), the European Research Council (Starting Grant No. 278203 WiQOJo), and the Agence Nationale de la Recherche (Grant JosePhSCharLi). A. G. and M. H. designed the sample and built the initial experiment based on an idea by M. H. and discussions with F. P. and E. D. F. A. G., S. J., D. H., F. G., and J. L. T. brought up the fabrication process. A. G., F. G., and J. L. T. built the sample. A. G. performed initial measurements and data analysis. F. B. and R. A. improved the measurement setup, data taking, and calibration routines. F. B. took final data and performed final data analysis. J. L. provided theory support. A. G. wrote the paper with figures from F. B. and input from all authors. 
[1] C. Padurariu, F. Hassler, and Y. V. Nazarov, Statistics of Radiation at Josephson Parametric Resonance, Phys. Rev. B 86, 054514 (2012).

[2] A. D. Armour, M. P. Blencowe, E. Brahimi, and A. J. Rimberg, Universal Quantum Fluctuations of a Cavity Mode Driven by a Josephson Junction, Phys. Rev. Lett. 111, 247001 (2013).

[3] V. Gramich, B. Kubala, S. Rohrer, and J. Ankerhold, From Coulomb-Blockade to Nonlinear Quantum Dynamics in a Superconducting Circuit with a Resonator, Phys. Rev. Lett. 111, 247002 (2013).

[4] S. Dambach, B. Kubala, V. Gramich, and J. Ankerhold, Time-Resolved Statistics of Nonclassical Light in Josephson Photonics, Phys. Rev. B 92, 054508 (2015).

[5] A. Grimm, Josephson Photonics: Statistics of Photons Emitted by Inelastic Cooper Pair Tunneling, Ph.D. thesis, Grenoble University (2015).

[6] S. Jebari, F. Blanchet, A. Grimm, D. Hazra, R. Albert, P. Joyez, D. Vion, D. Esteve, F. Portier, and M. Hofheinz, Near-Quantum-Limited Amplification from Inelastic Cooper Pair Tunneling, Nature Electronics 1, 223 (2018).

[7] F. Chen, J. Li, A. D. Armour, E. Brahimi, J. Stettenheim, A. J. Sirois, R. W. Simmonds, M. P. Blencowe, and A. J. Rimberg, Realization of a Single-Cooper-Pair Josephson Laser, Phys. Rev. B 90, 020506 (2014).

[8] M. C. Cassidy, A. Bruno, S. Rubbert, M. Irfan, J. Kammhuber, R. N. Schouten, A. R. Akhmerov, and L. P. Kouwenhoven, Demonstration of an AC Josephson Junction Laser, Science 355, 939 (2017).

[9] G.-L. Ingold and Y. V. Nazarov, Charge Tunneling Rates in Ultrasmall Junctions, in Single Charge Tunneling, NATO ASI Series (Springer, Boston, MA, 1992), pp. 21-107.

[10] T. Holst, D. Esteve, C. Urbina, and M. H. Devoret, Effect of a Transmission Line Resonator on a Small Capacitance Tunnel Junction, Phys. Rev. Lett. 73, 3455 (1994).

[11] M. Hofheinz, F. Portier, Q. Baudouin, P. Joyez, D. Vion, P. Bertet, P. Roche, and D. Esteve, Bright Side of the Coulomb Blockade, Phys. Rev. Lett. 106, 217005 (2011).

[12] Y. V. Nazarov and J. Danon, Advanced Quantum Mechanics: A Practical Guide (Cambridge University, Cambridge, United Kingdom, 2013).

[13] P. Delsing, K. K. Likharev, L. S. Kuzmin, and T. Claeson, Effect of High-Frequency Electrodynamic Environment on the Single-Electron Tunneling in Ultrasmall Junctions, Phys. Rev. Lett. 63, 1180 (1989).

[14] A. N. Cleland, J. M. Schmidt, and J. Clarke, Charge Fluctuations in Small-Capacitance Junctions, Phys. Rev. Lett. 64, 1565 (1990).

[15] M. H. Devoret, D. Esteve, H. Grabert, G.-L. Ingold, H. Pothier, and C. Urbina, Effect of the Electromagnetic Environment on the Coulomb Blockade in Ultrasmall Tunnel Junctions, Phys. Rev. Lett. 64, 1824 (1990).

[16] M. Westig, B. Kubala, O. Parlavecchio, Y. Mukharsky, C. Altimiras, P. Joyez, D. Vion, P. Roche, D. Esteve, M. Hofheinz, M. Trif, P. Simon, J. Ankerhold, and F. Portier, Emission of Nonclassical Radiation by Inelastic Cooper Pair Tunneling, Phys. Rev. Lett. 119, 137001 (2017).
[17] C. Rolland, A. Peugeot, S. Dambach, M. Westig, B. Kubala, C. Altimiras, H. le Sueur, P. Joyez, D. Vion, P. Roche, D. Esteve, J. Ankerhold, and F. Portier, Antibunched Photons Emitted by a DC Biased Josephson Junction, arXiv: 1810.06217.

[18] A. Grimm, S. Jebari, D. Hazra, F. Blanchet, F. Gustavo, J.-L. Thomassin, and M. Hofheinz, A Self-Aligned Nano-Fabrication Process for Vertical NbN-MgO-NbN Josephson Junctions, Supercond. Sci. Technol. 30, 105002 (2017).

[19] See Supplemental Material at http://link.aps.org/ supplemental/10.1103/PhysRevX.9.021016 for details on sample fabrication, sample design, extraction of sample parameters, measurement setup, algorithms, and calibration.

[20] M. P. da Silva, D. Bozyigit, A. Wallraff, and A. Blais, Schemes for the Observation of Photon Correlation Functions in Circuit QED with Linear Detectors, Phys. Rev. A 82, 043804 (2010).

[21] C. Eichler, D. Bozyigit, and A. Wallraff, Characterizing Quantum Microwave Radiation and Its Entanglement with Superconducting Qubits Using Linear Detectors, Phys. Rev. A 86, 032106 (2012).

[22] C. Negri and F. Pistolesi, Charge Fluctuations in SingleElectron Tunneling Oscillations, Phys. Rev. B 85, 115416 (2012).

[23] K. K. Likharev, Correlated Discrete Transfer of Single Electrons in Ultrasmall Tunnel Junctions, IBM J. Res. Dev. 32, 144 (1988).

[24] H. Vora, R. L. Kautz, S. W. Nam, and J. Aumentado, Modeling Bloch Oscillations in Nanoscale Josephson Junctions, Phys. Rev. B 96, 054505 (2017).

[25] S. Corlevi, W. Guichard, F. W. J. Hekking, and D. B. Haviland, Phase-Charge Duality of a Josephson Junction in a Fluctuating Electromagnetic Environment, Phys. Rev. Lett. 97, 096802 (2006).

[26] R. J. Glauber, Coherent and Incoherent States of the Radiation Field, Phys. Rev. 131, 2766 (1963).

[27] J. Leppäkangas, M. Fogelström, A. Grimm, M. Hofheinz, M. Marthaler, and G. Johansson, Antibunched Photons from Inelastic Cooper-Pair Tunneling, Phys. Rev. Lett. 115, 027004 (2015).

[28] J. Leppäkangas, M. Fogelström, M. Marthaler, and G. Johansson, Correlated Cooper Pair Transport and Microwave Photon Emission in the Dynamical Coulomb Blockade, Phys. Rev. B 93, 014506 (2016).

[29] A. A. Houck, D. I. Schuster, J. M. Gambetta, J. A. Schreier, B. R. Johnson, J. M. Chow, L. Frunzio, J. Majer, M. H. Devoret, S. M. Girvin, and R. J. Schoelkopf, Generating Single Microwave Photons in a Circuit, Nature (London) 449, 328 (2007).

[30] I.-C. Hoi, T. Palomaki, J. Lindkvist, G. Johansson, P. Delsing, and C.M. Wilson, Generation of Nonclassical Microwave States Using an Artificial Atom in 1D Open Space, Phys. Rev. Lett. 108, 263601 (2012).

[31] Y. Yin, Y. Chen, D. Sank, P. J. J. O’Malley, T. C. White, R. Barends, J. Kelly, E. Lucero, M. Mariantoni, A. Megrant, C. Neill, A. Vainsencher, J. Wenner, A. N. Korotkov, A. N. Cleland, and J. M. Martinis, Catch and Release of Microwave Photon States, Phys. Rev. Lett. 110, 107001 (2013). 
[32] M. Pechal, L. Huthmacher, C. Eichler, S. Zeytinoğlu, A. A. Abdumalikov, S. Berger, A. Wallraff, and S. Filipp, Microwave-Controlled Generation of Shaped Single Photons in Circuit Quantum Electrodynamics, Phys. Rev. X 4, 041010 (2014).

[33] B. Lounis and M. Orrit, Single-Photon Sources, Rep. Prog. Phys. 68, 1129 (2005).
[34] M. D. Eisaman, J. Fan, A. Migdall, and S. V. Polyakov, Single-Photon Sources and Detectors, Rev. Sci. Instrum. 82, 071101 (2011).

[35] E. Knill, R. Laflamme, and G. J. Milburn, A Scheme for Efficient Quantum Computation with Linear Optics, Nature (London) 409, 46 (2001). 